BNL - 67337

CAP-263-Muon-00R

Informal Report

MEASUREMENTS OF THE PROPERTIES OF BERYLLIUM FOIL

Yongxiang Zhao and Haipeng Wang

Brookhaven National Laboratory

March 2000 


\title{
MEASUREMENTS OF THE PROPERTIES OF BERYLLIUM FOIL
}

\author{
Yongxiang Zhao, Haipeng Wang \\ Physics Department, Bldg. 901-A \\ Brookhaven National Laboratory, Upton, NY 11973
}

\begin{abstract}
.
The electrical conductivity of beryllium at radio frequency $(800 \mathrm{MHz})$ and liquid nitrogen temperature were investigated and measured. This summary addresses a collection of beryllium properties in the literature, an analysis of the anomalous skin effect, the test model, the experimental setup and improvements, MAFLA simulations, the measurement results and data analyses. The final results show that the conductivity of beryllium is not as good as indicated by the handbook, yet very close to copper at liquid nitrogen temperature.
\end{abstract}

\section{INTRODUCTION}

A muon collider requires rapid acceleration, and thus a huge amount of RF power. ${ }^{[1]}$ The preference is to approach highest gradient and lowest RF power requirement. In order to reduce $\mathrm{RF}$ power it is considered to apply liquid nitrogen cooling so as to reduce the metallic resistivity ${ }^{[2]}$. We found from the handbook ${ }^{[3]}$ that at liquid nitrogen temperature beryllium has the best conductivity of all metals, though at room temperature it is poorer than copper. The approach for highest possible gradient leads to the application of pill-box cavity, of which the maximum surface electric field equals to the acceleration field. But it requires a window, which closes the electromagnetic field while allowing the muon to pass through. Beryllium is the structural metal with the lowest atomic number, so that it is the best candidate for a window to minimize scattering of the muon beam.

Therefore, beryllium operated in liquid nitrogen temperature is very interesting. However, the data on handbook are for highly pure beryllium. Moreover, the data disperse in a wide range in different experimental record ${ }^{[4]}$. To this end, it is considered necessary to check the real conductivity of beryllium.

An experiment to measure the resistivity of beryllium at liquid nitrogen has been done. It shows the conductivity is not as good as indicated on handbook, yet is almost the same as that of copper at LN temperature.

This note is a summary of the investigation of the electrical conductivity of beryllium and a few of its alloys. A few samples were tested. They include Be IF-1, Be-Al alloy AlBeMet162, AlBeMet150 from BrushWellman, Be from ESPI. All material is in foil form. 


\section{THE PROPERTIES OF BERYLLIUM IN LITERATURE}

Fig. 1 shows the dc resistivity of beryllium at low temperature in comparison with copper ${ }^{1}$. One can see that beryllium is better in the range from $50 \mathrm{~K}$ to $150 \mathrm{~K}$. However, the data quoted is for very pure material. In different reports, the data spread widely, as shown in Fig. 2, quoted from reference [4]. More details can be found from reference [5].

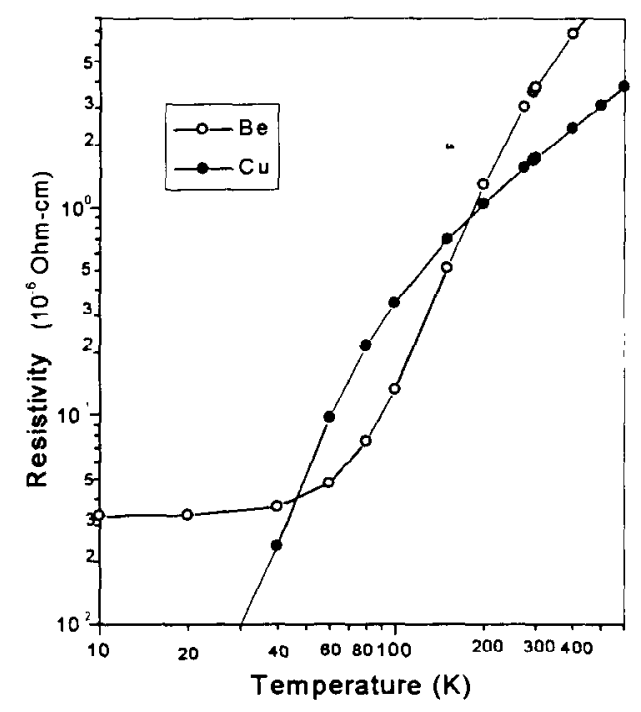

Fig. 1 The resistivity of $\mathrm{Be}$ and $\mathrm{Cu}$

Fig. 3 shows the thermal conductivity of beryllium from different sources ${ }^{[6,7]}$. Also shown is that of copper for comparison ${ }^{[3]}$. The two curves for beryllium also show considerable variation. Nevertheless, in the vicinity of liquid nitrogen temperature, they all show thermal conductivity better than copper. This suggests that its electrical conductivity, too, might be better.

Since the data strongly depend on the purity of the sample, these curves can be only suggestive.

For purposes of completeness, Fig. 4 also collects other properties of beryllium from variety sources.

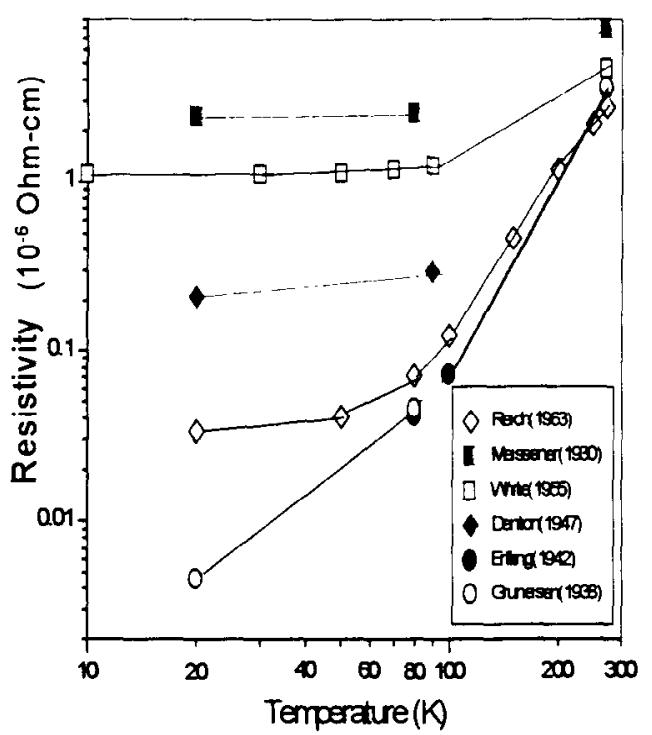

Fig. 2 The resistivity of $\mathrm{Be}$ from different experiments.

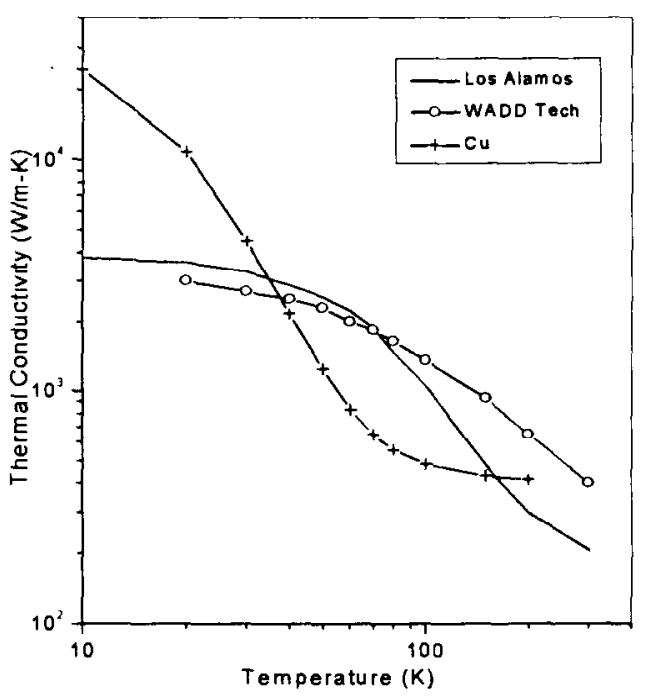

Fig. 3 Thermal conductivity of beryllium

\footnotetext{
${ }^{1}$ The data were quoted from [3]. Note that there is a typo at $60 \mathrm{~K}$, which has been checked with its original source and corrected here on Fig. 1.
} 

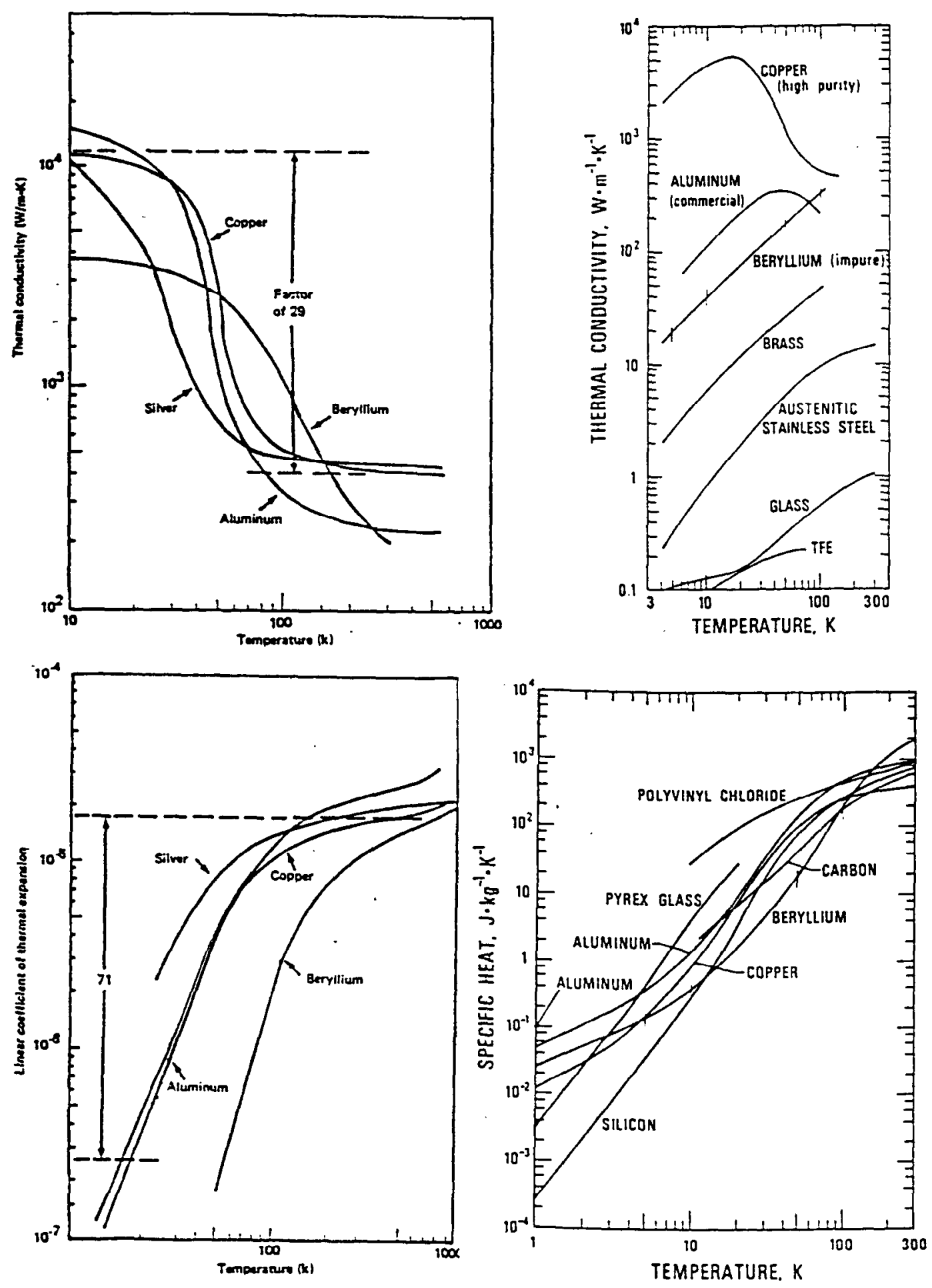

Fig. 4 Other properties of beryllium. (a) (b) Thermal conductivity from different sources; (c)Coefficient of thermal expansion; (d) Specific heat. 


\section{THE ANOMALOUS SKIN EFFECT}

It is known that for most metals, the cooler the temperature, the higher the dc electric conductivity. The experimental enhancement factor of dc conductivity usually characterized by RRR (residual resistivity ratio) at liquid helium temperature or just before converting to superconductivity. For copper RRR is $50-200$. However, for high frequency, the metallic surface conductivity approaches to a constant due to anomalous skin effect. For example, for copper at liquid helium temperature, $4 \mathrm{~K}$, its surface resistance is about only 4 times better than that at room temperature (at frequency of $10 \mathrm{GHz}$ ) ${ }^{[8]}$, or $\mathrm{Q}$ is 4 times higher, though according to classical theory it would be $7 \sim 14$ times better ${ }^{1}$. Some other experimental results also indicated the $\mathrm{Q}$ enhancement at low temperature is less than $4\left(3.77\right.$ at $432 \mathrm{MHz}, 20 \mathrm{~K}^{[9]}, 3$ to 3.6 at 0.96 to $7 \mathrm{GHz}, 4 \mathrm{~K}^{[10]}$ ).

Our intention is to apply beryllium foil as a window in a cavity, but we lack RF data. Therefore the anomalous skin effect is very much of concern. The question is if the conductivity improvement under cryogenic condition will be offset.

In order to answer this question, let's review the anomalous effect. Based on the free electron theory, the dc electric conductivity $\sigma$ can be deduced to be:

$$
\sigma=\frac{n e^{2} \tau}{m}=\frac{n e^{2} l}{m v_{0}}
$$

where $n$ is the concentration of the electrons, $e$ and $\mathrm{m}$ the electron charge and mass, $\tau$ the relaxation time or the mean time between two collisions, $l$ the mean free path of the electrons and $v_{0}$ the Fermi velocity, which is of the order of $10^{6} \mathrm{~m} / \mathrm{s}{ }^{[8]}$. The skin depth is:

$$
\delta_{k}=\sqrt{2 / \omega \mu \sigma} .
$$

The distinction between normal and anomalous skin effect is illustrated in Fig. 5. The external electromagnetic field penetrates to the metal surface and will be faint beyond the skin

A

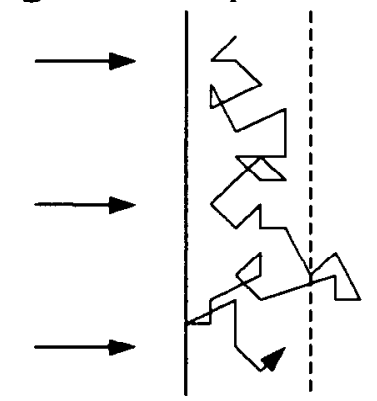

B

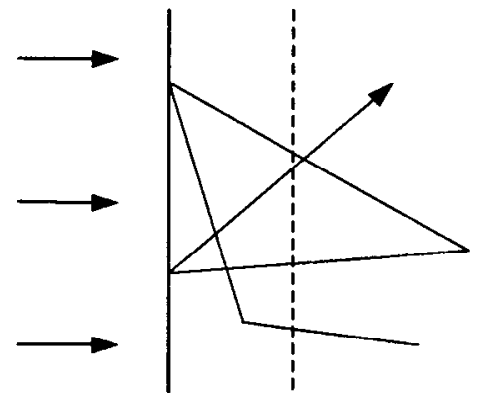

Fig. 5 The trace of electron inside the metal; (A) normal case; (B) anomalous case.

The solid line is the surface, dashed line denotes the skin depth.

depth layer. Normally, the free path of electron is less than the skin depth (Fig.5-A, $\delta>>$ ), while in the anomalous case, it is larger than the skin depth (Fig.5-B, $\delta<<l$ ). In the later case, an electron will be subject to the field for only a small part of its transit time between two collisions.

\footnotetext{
${ }^{1}$ Casmir ${ }^{[8]}$ suggests the very pure copper has its conductivity $10^{4}$ times better at liquid helium temperature, and thus $Q$ will be 100 times better.
} 
Consequently, it is equivalent to a lesser electron concentration in the skin layer, and that causes poorer conductivity. The anomalous skin depth is approximately ${ }^{[8]}$ :

$$
\delta^{3}=\left(\frac{2}{b}\right)\left(\frac{v}{\omega}\right) \lambda_{p}^{2},
$$

where $\lambda_{p}{ }^{2}=m / \mu m_{e} e^{2}$ is the plasma wavelength. If the anomalous effect occurs, the skin depth will be larger than that calculated from the classical formula (2).

In our case, assuming $f=800 \mathrm{MHz}$, the resistivity of beryllium is $0.133 \times 10^{-8} \Omega \mathrm{m}$ (from handbook ${ }^{[3]}$ at $100 \mathrm{~K}$ ). For standard metal $n_{e}=6.0 \times 10^{28} \mathrm{~m}^{3}, l \sigma=8.3 \times 10^{-16} \Omega-\mathrm{m}^{2}$, and $\lambda_{p}=$ $2.1810^{-8} \mathrm{~m}, b=7.3$, then we get

normal skin depth

$$
\begin{array}{ll}
\text { normal skin depth } & \delta_{k}=0.64 \mu \mathrm{m}, \quad \text { mean free path } l=0.62+\mu \mathrm{m} \\
\text { anomalous skin depth } & \delta=0.33 \mu \mathrm{m} .
\end{array}
$$

These data are of the same order, but the anomalous skin depth is smaller. It suggests that the anomalous effect is not obvious.

We may also check the significance of anomalous effect by Pippard's curve ${ }^{[1]}$ shown in Fig.6, which is based on the theory of Reuter and Sondheimer ${ }^{[12]}$. The vertical ordinate is $R_{\infty}, R$, where $R$ is surface resistance, $R_{\infty}$ refers to extreme anomalous case. The abscissas $\alpha^{l, 6}$, where

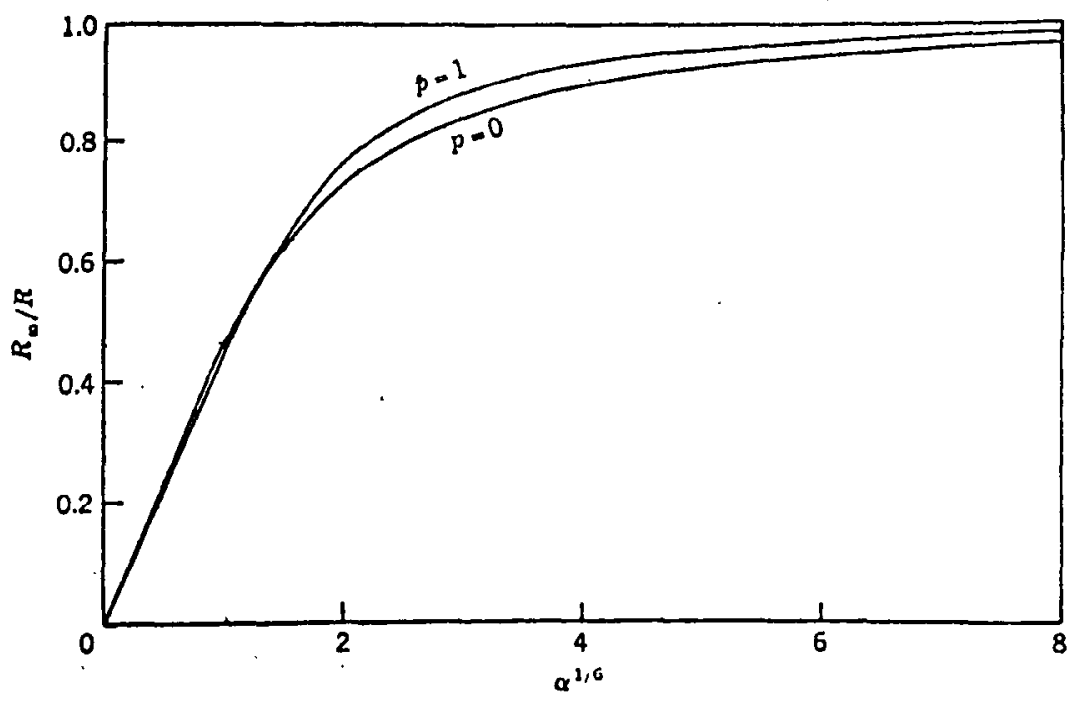

Fig. $6 R_{\infty} / R$ vs $\alpha^{l / 6}$ for $p=0$ and 1

$\alpha=3 \pi \omega \sigma l^{2}=\frac{3}{2} l^{2} / \delta^{2}$ and $\alpha^{1 / 6} \propto \sqrt{\sigma}$. The ordinate is so chosen that it has the merit of giving a straight line in the small $\alpha$, which corresponds to the classical limit. The parameter $p$ on the plot is a measure of electron scattered from the metal surface when hitting from internally. A fraction $p$ of electrons is specularly reflected, the remaining fraction (1-p) being diffusely scattered. $p=0$ and $p=I$ are two extreme cases. Experiment demonstrated most cases close to $p$ $=0$. 
This curve shows that the resistivity offsets straight line only if $\alpha^{1 / 6}>1.5$. For the above data set, we get $\alpha^{1 / 6}=1.056$, which suggests the resistance still falls in the normal region.

Therefore, we can conclude that beryllium at liquid nitrogen temperature and $800 \mathrm{MHz}$ evidences classical, not anomalous, resistivity.

\section{THE TEST MODEL}

Our purpose is to test the conductivity of beryllium at liquid nitrogen temperature and at a specified frequency. The material must be commercially available in a form suitable for our needs. Thus the test sample should meet the following requirements.

(1) The purity is industrially achievable.

(2) It is a foil with thickness of 5 mils or so, which is allowable for a window without too much scattering.

To test RF conductivity directly is not easy. We chose instead to measure the $\mathrm{Q}$ of a resonator, our goal being to know the $\mathrm{Q}$ factor enhancement at liquid nitrogen temperature. Therefore, measuring $Q$ directly is, of course, appropriate.

To this end, a tape resonator, which can meet our need and be easy to build, is preferable. This resonator is simply formed by two parallel tapes with a length of half wavelength. The field is of TEM-mode, for which the field pattern is simply a sine wave. The advantages of this model are:

(1) No conduction loss, which is usually uncertain and causes test error.

(2) The tape can be of any thickness to satisfy our final application.

(3) The field is concentrated in the region between two tapes; consequently the environment will not perturb $Q$ significantly.

The disadvantages are:

(1) Dielectric support is necessary; this causes extra loss.

(2) The magnetic field at the edges of the tape is logarithmically singular, since the curvature radius is zero, so it is difficult to determined the loss, also makes difficult any absolutevalue test; only a relative-value test is reliable.

Two kinds of beryllium samples were purchased. One is from BrushWellman, and another is from ESPI. Later we also tested two Be-Al alloy foils, AlBeMet150 and AlBeMet162, made by BrushWellman. All samples were of the same size, $0.8 \mathrm{in} \times 6.25 \mathrm{in} \times 0.006 \mathrm{in}$. Table 1 lists their major grads and costs.

Table 1

\begin{tabular}{|l|l|l|c|c|l|}
\hline Material & \multicolumn{1}{|c|}{ Company } & Factory id & Grad & Real Purity & Cost / ea \\
\hline $\mathrm{Be}$ & BrushWellman & IF-1 & $99.8 \%$ & Be $99.89 \%$ & $\$ 623$ \\
\hline $\mathrm{Be}$ & ESPI & NI 120 & $99.9 \%$ & & $\$ 1140$ \\
\hline Be alloy & BrushWellman & AlBemet150 & & Be $50 \%$ & $\$ 207$ \\
\hline Be alloy & BrushWellman & AlBemet162 & & Be $62 \%$ & $\$ 215$ \\
\hline
\end{tabular}




\section{THE EXPERIMENTAL SETUP AND IMPROVEMENTS}

Fig. 7 shows the cryogenic tank with its RF cavity. The beryllium test model assembly (not shown) was put inside a copper cavity. The reason for putting test model inside a cavity is to avoid radiation loss, because a tape resonator is an open structure, although its energy is mainly concentrated near the tapes. The cavity itself can also be used to test the $Q$ enhancement of copper and serve for the support of the RF energy couplers ${ }^{1}$.

The major dimensions of the tapes, cavity and cryogenic tank are as follows:

Tape

Tape gap

Cavity

Cryo tank
0.8 "W $\times 6.25$ "L $\times 0.006$ "D $(20.3 \mathrm{~mm} \times 158.7 \mathrm{~mm} \times 0.15 \mathrm{~mm})$ with 2 holes (dia 0.125 ") at two ends in a distance of 0.2 " $0.25^{\prime \prime}(6.35 \mathrm{~mm})$, between two tapes, see Fig. 9 Inner diameter 11.25" (286 mm), Inner Height 3.94" (100 mm) Inner diameter $\sim 20$ ", inner height $\sim 22$ "

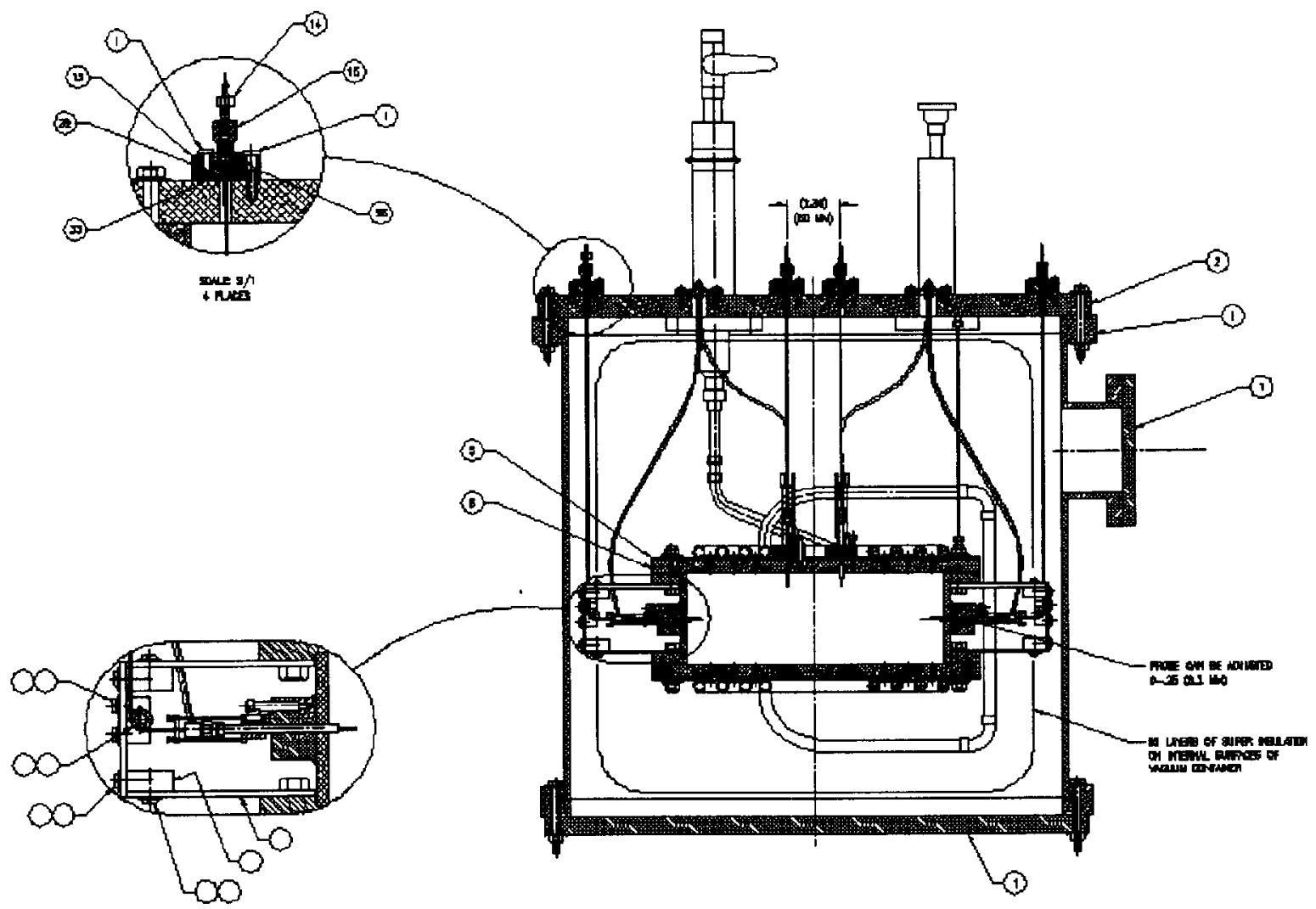

Fig. 7 The cryogenic tank assembly

Although the principle is simple, we encountered a lot of technical problems. Among them are cryogenic problems, errors caused by dielectric loss, mechanical instability and the accuracy of temperature measurement ${ }^{[14]}$. Here we review our progressive improvements.

The insulation of the cryogenic tank was adequate. Since the beryllium tapes were suspended inside the cavity with dielectric supports, the $\mathrm{LN}_{2}$ flow cools only to the cavity, the

\footnotetext{
${ }^{1}$ The copper test result has been reported earlier, see [13].
} 
thermal balance was very slow. In order to speed up the cooling of the tape, we injected a bit helium gas after the cavity reached $\mathrm{LN}_{2}$ temperature. Warm-up also was very slow. To speed up warm-up, we disconnected the cooling pipe from liquid nitrogen buggy and connected it to pressurized air pipeline. The forced air at room temperature is adequate for warming up.

Although Rexolite ${ }^{\mathrm{TM}}$ is the best commercially available dielectric, with the lowest $\mathrm{rf}$ loss, the influence on $Q$ factor was still substantial. In order to reduce the dielectric loss, a few methods were tried ${ }^{[14]}$. The lowest loss was obtained by applying a central support, leaving both ends free. The center of the tape is at the node of electric field causing little loss. However, with free ends we found that the gap was nonuniform, the mechanical repeatability was poor, and so was the frequency. We tried to use two Teflon ${ }^{\mathrm{TM}}$ tapes wrapping the beryllium tapes and stretched both ends that the Be tapes was suspended with very little volume of dielectric attaching it. The loss was light but encountered the same problem of mechanical stability.

The final beryllium test model assembly is shown in Fig.9 (schematic). The Be tapes were held by two Rexolite clips, which were held in tension by screws situated far from the tapes, where the electric field is very weak. Note that the clip is caved-in near the tape end, where the electric field is the maximum. This reduces the dielectric loss. The spacer between the two tapes in the original design was a Rexolite block (option 2 in Fig.9). The experiment showed a substantial loss due to the spacer, so we modified the spacer to pin shape (option 1), which minimizes the volume.

In order to improve the mechanical stability, a pedestal, on which the clips were embedded so as to prevent tape twist, was added on the assembly. Another modification was to maintain constant tension despite different expansion coefficients. An experiment of frequency dependence on the tensioning force showed a rate of $1.86 \mathrm{MHz} / \mathrm{kgf}$ for the tape TEM mode (see Fig. 8). Therefore we added a weight and a balance to one screw, and mounted outside the cavity so as to maintain constant tension ${ }^{[14]}$.

At first, four thermal sensors were mounted only on the cavity,

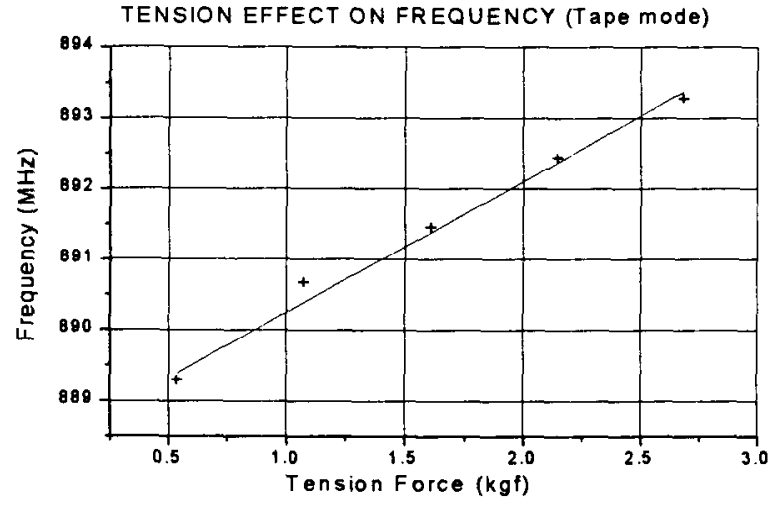

Fig. 8 The effect of tension on frequency believing that any lead put inside the cavity would perturb the field pattern and thus cause error. The tape temperature was not monitored directly, but relied on thermal equilibration. However, equilibration was very slow. Moreover, the tape was not in thermal equilibrium during cooldown and warm-up. We found that direct monitoring was necessary. Trying to glue a sensor on the tape failed. Eventually, we used another clip to enforce the contact between the sensor and the tape. The sensor is seated at the center, the node of electric field, so that it causes little perturbation. The lead of the sensor has to be laid out carefully, either along the central axis or attached the bottom metal surface. In both cases no electric field is parallel with the lead. Experimentally it had little influence on either the frequency or the $\mathrm{Q}$ factor. 


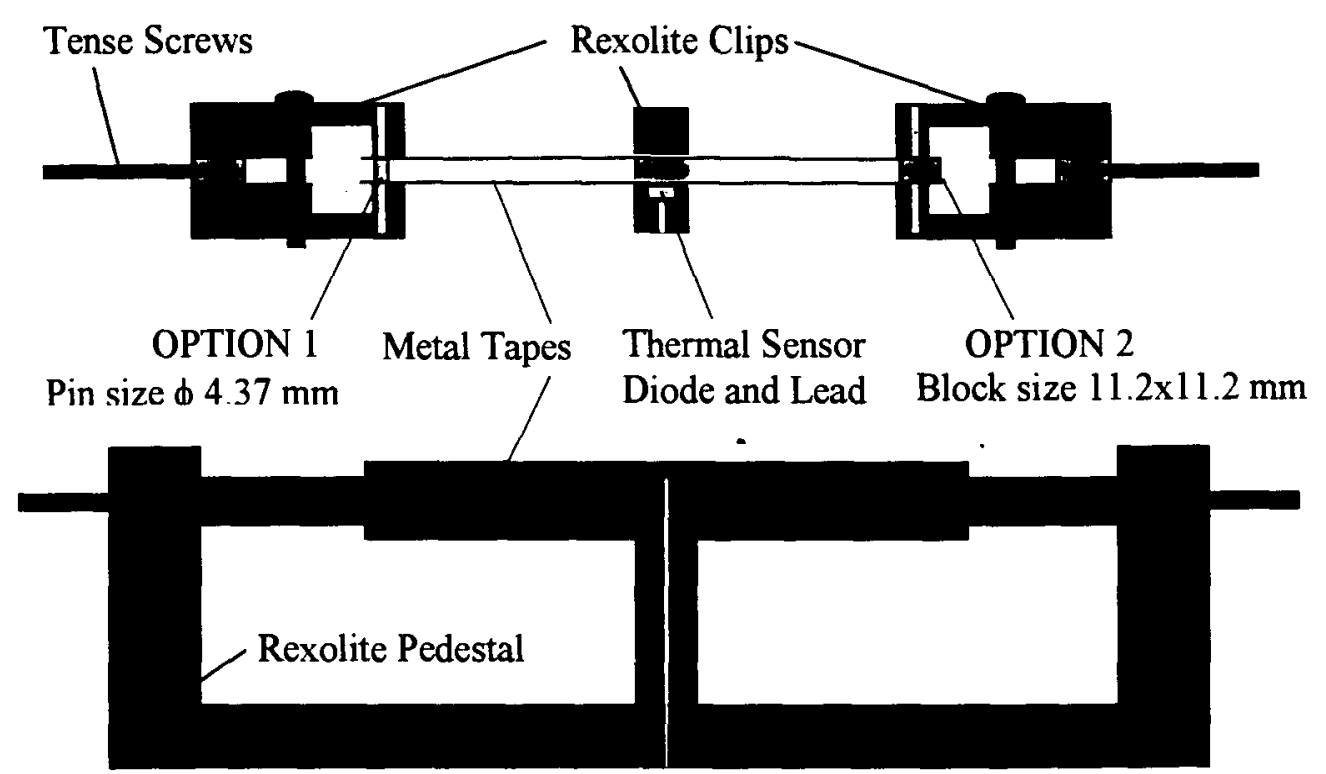

Fig. 9 The final beryllium test model assembly

\section{MAFIA SIMULATION}

The purposes of MAFIA simulation were threefold. (1) Check the frequency and field pattern as well as other possible modes. (2) Check the $Q$ factor. (3) Check the dielectric losses so as to optimize the shape of the supporter.

It was found there are three modes below $1 \mathrm{GHz}$. One is the tape mode, which is what we want to test. The field pattern, a TEM. mode with sine wave distribution shown in Fig. 10, is exactly as expected. Shown in Fig. 11 is the cavity mode TM100, in which the field pattern changes a little due to introducing the tape assembly, while with a sizable drop in resonant frequency. Shown in Fig. 12 is another cavity mode, the deformed TM110 mode, which is perturbed seriously due to the tape. All these three modes were observed in our experiment, reasonably coincident with the simulation.

The $Q$ factor obtained by our MAFIA simulation was much higher than that from measurement. At first we attributed the difference to coarse mesh which we used. A fine mesh of a 3D program would have prolonged the CPU time excessively. Later on we realized that the problem arises from the edge of the tape. Our first simulation assumed a tape thickness of zero. A zero radius of curvature of the edge implies an infinite local current density, which in turn may cause infinite loss. The current is mainly distributed on the center part of the tape, where the field pattern is the same as in a TEM mode. A TEM mode can be dealt with as a $2 \mathrm{D}$ problem, which requires many fewer mesh points. Therefore we can estimate how much error could be introduced due to a coarse mesh. 
Fig. 10

Field Pattern of Tape Mode

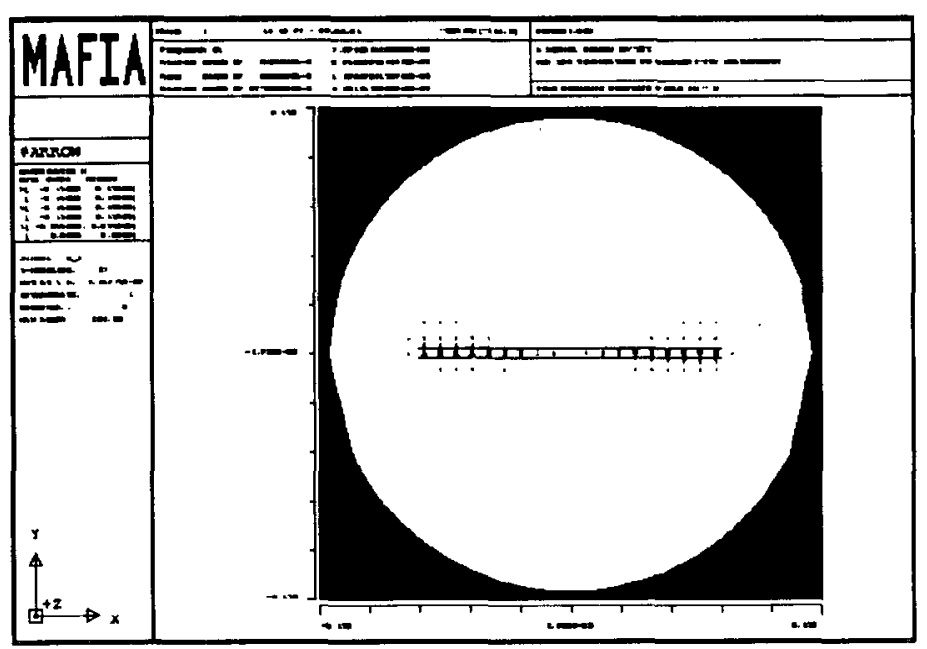

Fig. 11

Field Pattern of TM100 Cavity Mode

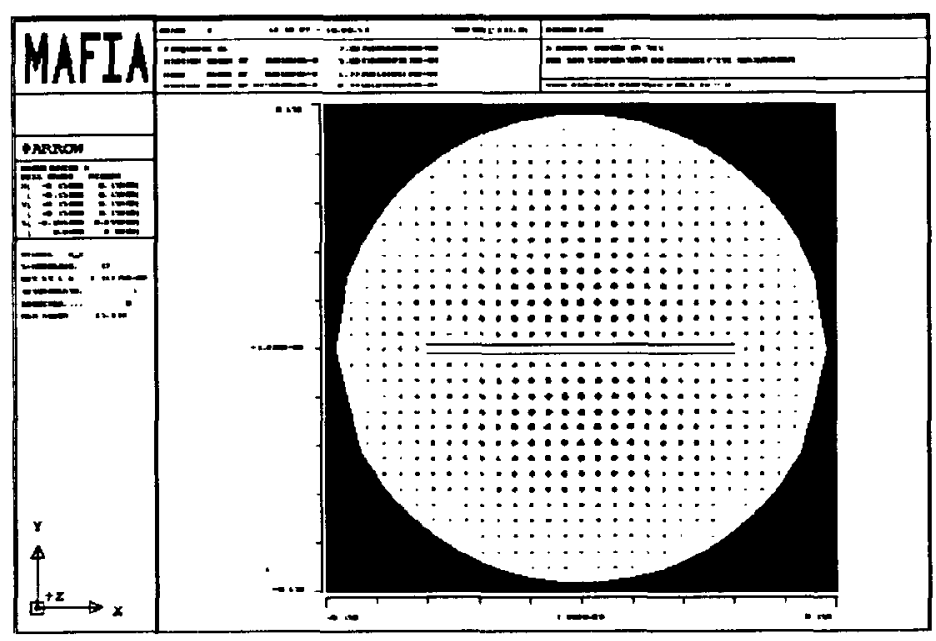

Fig. 12

Field Pattern of Deformed TM110 Cavity Mode

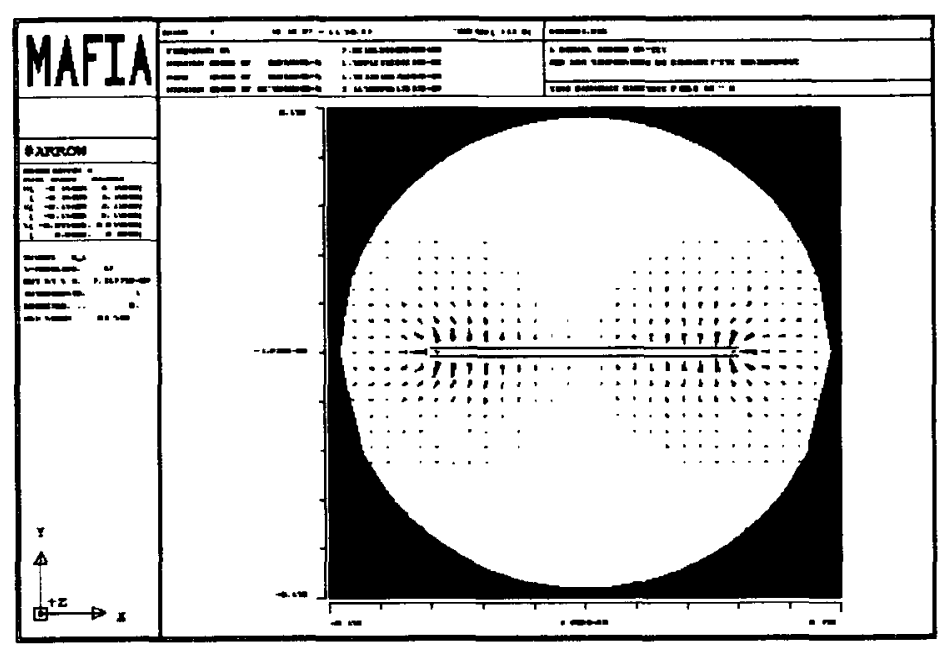


Therefore we ran a series of MAFIA 2D cases. The following table summarizes the results, with tape of a finite thickness. The third column shows the number of mesh points. The 6th column indicates the corresponding $Q$ factor.

Table 2

\begin{tabular}{|c|c|c|c|c|c|c|c|}
\hline Program & $\begin{array}{c}\text { Tape } \\
\text { Thickness }\end{array}$ & $\begin{array}{c}\text { Total Mesh } \\
\text { Points }\end{array}$ & $\begin{array}{c}\text { Auto- } \\
\text { mesh } 1\end{array}$ & $\begin{array}{c}\text { \# of inner } \\
\text { Lines }^{2}\end{array}$ & $Q$ & $\begin{array}{c}Q \\
\text { Ratio }\end{array}$ & Accuracy \\
\hline tp423 & $0(\mathrm{~mm})$ & 825 & no & 4 & 3380 & $100 \%$ & $0.58 \%$ \\
\hline tp428a & 0.15 & 5112 & yes & 4 & 3356 & $99.3 \%$ & $2.2 \%$ \\
\hline tp428b & 0.15 & 20022 & yes & 8 & 3087 & $91.3 \%$ & $1.4 \%$ \\
\hline tp428c & 0.15 & 80089 & yes & 16 & 2875 & $85.1 \%$ & $4.5 \%$ \\
\hline tp428d & 0.15 & 107406 & yes & 32 & 2725 & $80.6 \%$ & $* 15 \%$ \\
\hline tp428e & 0.15 & 128304 & yes & 40 & 2070 & $61.1 \%$ & $* 26 \%$ \\
\hline
\end{tabular}

1. "Automesh" means the mesh is established automatically by MAFIA, usually nearly uniform

2. Number of mesh lines inside the tape gap (between tape and central axis)

3. "Accuracy" is a measure of the consistency of the computation with Maxwell equations, not the error of the result. The marker * indicates that the result is not precise.

As the mesh became finer, the MAFIA solution became more and more inconsistent with Maxwell's equations. Nevertheless, the penultimate column indicates that the $Q$ became more in agreement.

Why was the finer mesh non-Maxwellian? The cross section of the tape is rectangular, with a width of $0.15 \mathrm{~mm}$ and a length of $20.32 \mathrm{~mm}$. Although the thickness is finite, there are still two sharp corners. At the apex of the angle the radius of curvature is zero. Theoretically, field intensity will approach infinite if the curvature approaches zero. Mathematically, it is a singularity. So the field near the apex will change rapidly. A computer code deals with those discrete data in a way that has been somehow averaged between two mesh points so as to output a finite number. In a coarse mesh the data variations are smoothened, while in a fine mesh those data change too fast to converge. Then the code issues an accuracy warning. MAFIA cannot deal with geometries with sharp angles.

To this end, we turned to analytical method. A 2D problem (a static electric field, or a TEM mode) can be solved by conformal mapping. If the thickness of the tape is zero, the loss will be divergent $[15]$. For a finite thickness, the field at the corner still is infinite, but its integral is finite, and so is the loss. We found that the error of a coarse mesh could be as high as $60 \%$. This elucidates the discrepancy between the MAFIA predictions (with coarse mesh) and our experiment. [15]

As to the dielectric loss, we found its influence on $\mathrm{Q}$ factor is significant, higher than expected. However, the experiment cannot identify individual contributions from different components or parts. MAFIA is a powerful tool then. It also is helpful to trim the shape of the parts. Fig. 13 shows the final geometry of test setup. Parts 2 and 3 comprise only one component physically. We partitioned it into two parts by marking them with different "mat" number, to 


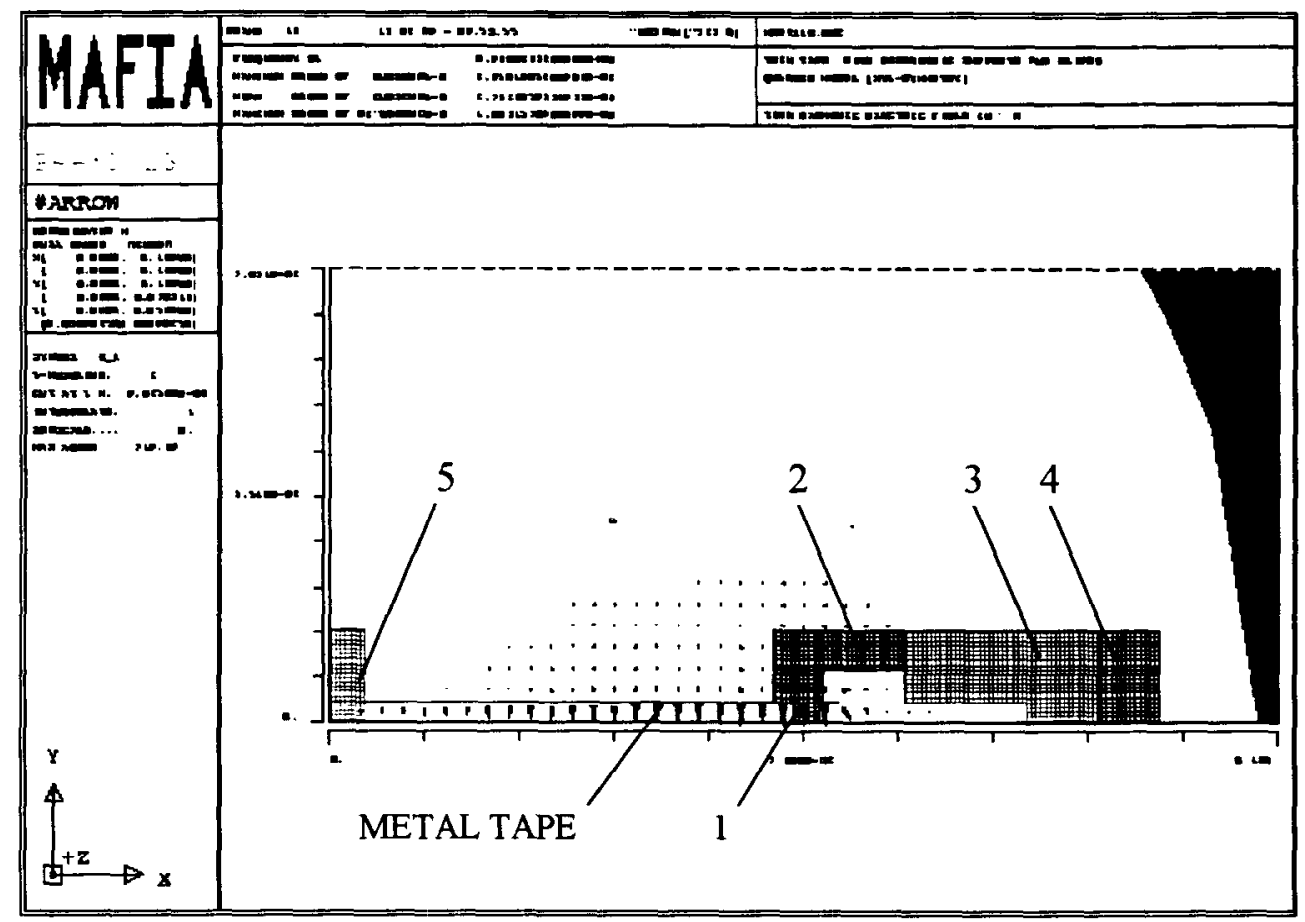

1. Spacer; 2. Clip-a, 3. Clip-b, 4. Pedestal, 5. Clip-c

Fig. 13 The geometry of test set up for MAFIA Simulation

which parameters of the material can be defined in the MAFIA program, in order to distinguish their contribution to the losses.

We also found that the spacer, used for defining the gap between the tapes, has a significant affection on the $Q$ factor, even though its size is very small. The two spacers, one a block and the other a pin, were calculated.

Tables 3 and 4 summarize the results. The dielectric loss angle is arbitrarily assumed to be 0.001 , which is not exactly the real case. The catalogue lists the loss angle of Rexolite as 0.0002 at $1 \mathrm{MHz}$ and room temperature ${ }^{[16]}$. The real loss angle at $800 \mathrm{MHz}$ has been figured out via experiment and will be addressed later. The metal loss is based on the MAFIA default assumption that the conductivity is $K=5.8 \times 10^{7} \mathrm{mho} / \mathrm{m}$. Note that these simulation were with a coarse mesh.

Table 3 Loss calculation with pin spacer

\begin{tabular}{|l|c|c|c|c|c|c|}
\hline & id \# & $\mathrm{f}[\mathrm{MHz}]$ & $\mathrm{Q}$ & Metal loss & Dielectric loss $^{1}$ & Loss ratio $^{2}$ \\
\hline Metal loss only & & 894.0825 & 3520 & $5.6525 \mathrm{E}-05$ & $0.0000 \mathrm{E}+00$ & 0.00 \\
\hline Spacer & 1 & 894.0825 & 3197 & $5.6525 \mathrm{E}-05$ & $5.7192 \mathrm{E}-06$ & 10.12 \\
\hline Clip-a & 2 & 894.0825 & 3266 & $5.6525 \mathrm{E}-05$ & $4.4025 \mathrm{E}-06$ & 7.79 \\
\hline Clip-b, -c & 3,5 & 894.0825 & 3498 & $5.6525 \mathrm{E}-05$ & $3.5347 \mathrm{E}-07$ & 0.63 \\
\hline Pedestal & 4 & 894.0825 & 3518 & $5.6525 \mathrm{E}-05$ & $4.1799 \mathrm{E}-08$ & 0.07 \\
\hline Total loss & & 894.0825 & 2968 & $5.6525 \mathrm{E}-05$ & $1.0517 \mathrm{E}-05$ & 18.61 \\
\hline
\end{tabular}


1. Assuming dielectric loss angle $=0.001 .2$. Loss ratio $=$ dielectric loss $/$ metal loss.

Table 4 Loss calculation with block spacer

\begin{tabular}{|l|c|c|c|c|c|c|}
\hline & id \# & $\mathrm{f}[\mathrm{MHz}]$ & $\mathrm{Q}$ & Metal loss & Dielectric loss & Loss ratio $^{2}$ \\
\hline Metal loss only & & 841.3181 & 3408 & $5.4946 \mathrm{E}-05$ & $0.0000 \mathrm{E}+00$ & 0.00 \\
\hline Spacer & 1 & 841.3181 & 1949 & $5.4946 \mathrm{E}-05$ & $4.1101 \mathrm{E}-05$ & 74.80 \\
\hline Clip-a & 2 & 841.3181 & 3193 & $5.4946 \mathrm{E}-05$ & $3.6934 \mathrm{E}-06$ & 6.72 \\
\hline Clip-b, -c & 3,5 & 841.3181 & 3389 & $5.4946 \mathrm{E}-05$ & $2.9736 \mathrm{E}-07$ & 0.54 \\
\hline Pedestal & 4 & 841.3181 & 3406 & $5.4946 \mathrm{E}-05$ & $3.5405 \mathrm{E}-08$ & 0.06 \\
\hline Total loss & & 841.3181 & 1871 & $5.4946 \mathrm{E}-05$ & $4.5127 \mathrm{E}-05$ & 82.13 \\
\hline
\end{tabular}

1. Assuming dielectric loss angle $=0.001$. 2. Loss ratio $=$ dielectric loss $/$ metal loss.

From the last column in the Tables one can conclude that the dielectric loss is dominated by the spacer. Especially for the block spacer case it reduces the Q significantly. Therefore we have to minimize the volume of spacer to the pin shape. The second important part is "clip-a (\#2)". This motivated the cut cavern near the tape edge. The remaining parts were demonstrated to be less important. Especially, it is acceptable to clip a thermal sensor directly on the center tape.

The frequencies calculated from MAFIA shown above are very close to those from measurements. Therefore, the dielectric constant of 2.5, assumed in the MAFIA simulation, is correct.

\section{THE RESULTS OF MEASUREMENT}

A number of experiments were done. Here we show some typical results. Fig. 14 shows the $\mathrm{Q}$ factor of beryllium, which increases along with the decreasing temperature. Also shown is that during warm-up. They are indistinguishable in the plot. Fig.15 shows the same performance, except that the spacer is a block instead of a pin. Clearly its Q factor is lower. Figs. 16 and Fig.17 show the similar performance for a copper tape with the same thickness.

Note that in Fig. 17 there exists a significant abnormality at temperature ranging from $160 \mathrm{~K}$ to $250 \mathrm{~K}$. This error is mainly due to modes interference. As mentioned above there are three modes. Table 5 lists the rough frequencies. Those frequencies vary within a few $\mathrm{MHz}$ with temperature and the geometry, which might vary slightly when reassembled.

Table 5 Frequencies of different mode

\begin{tabular}{|c|c|c|}
\hline \multirow{2}{*}{ Mode } & \multicolumn{2}{|c|}{ Spacer } \\
\cline { 2 - 3 } & Pin & Block \\
\hline Tape mode - TEM & $890 \mathrm{MHz}$ & $840 \mathrm{MHz}$ \\
\hline Cavity mode - TM110 & $798-818 \mathrm{MHz}$ & $807-823 \mathrm{MHz}$ \\
\hline Cavity mode - TM010 & $756 \mathrm{MHz}$ & $756 \mathrm{MHz}$ \\
\hline
\end{tabular}




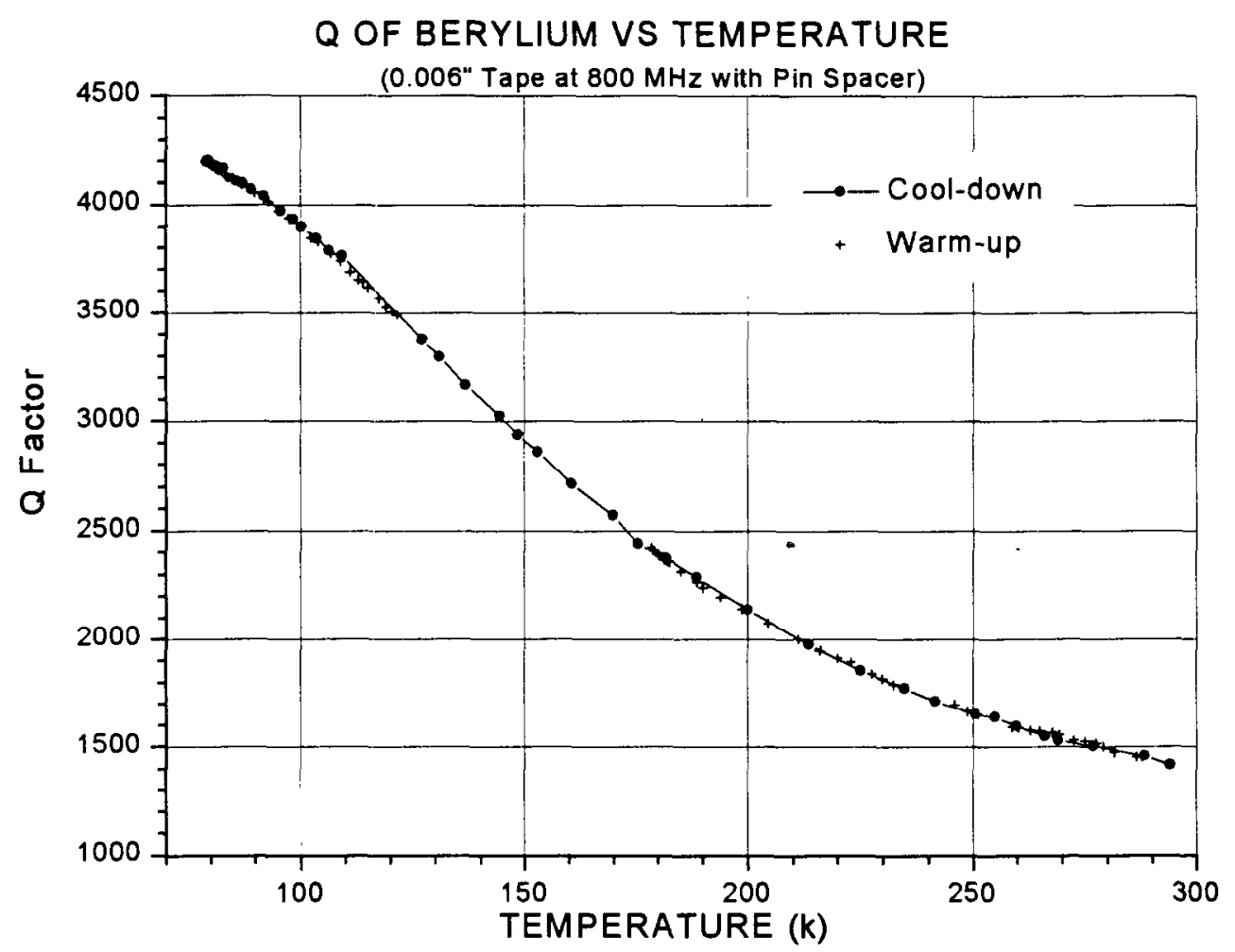

Fig. 14 Beryllium $Q$ factor (with pin spacer)

MU-workbe-explorignyan28G1

Q OF BERYLLIUM VS TEMPERATURE

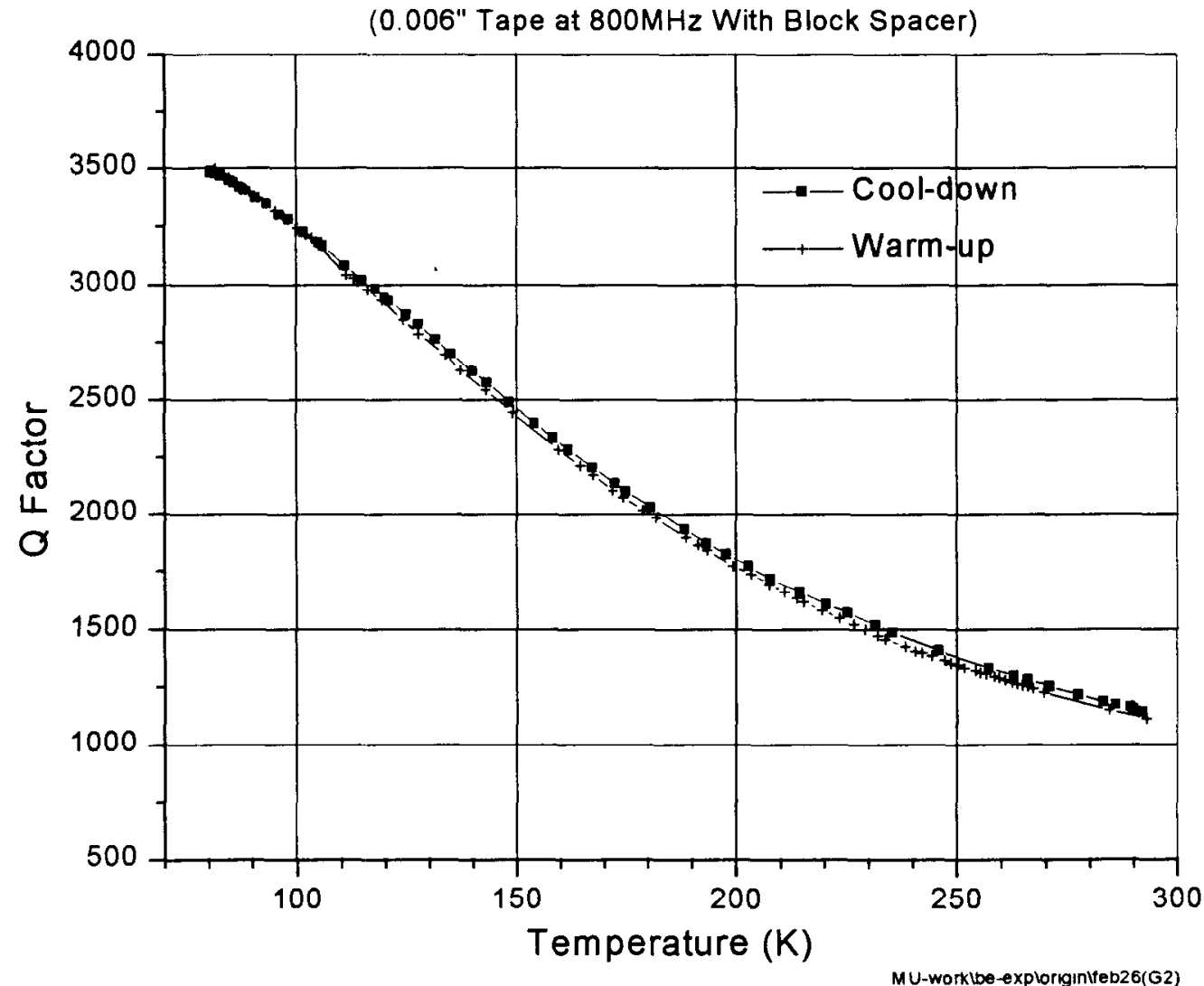

Fig. 15 Beryllium Q factor (with block spacer) 


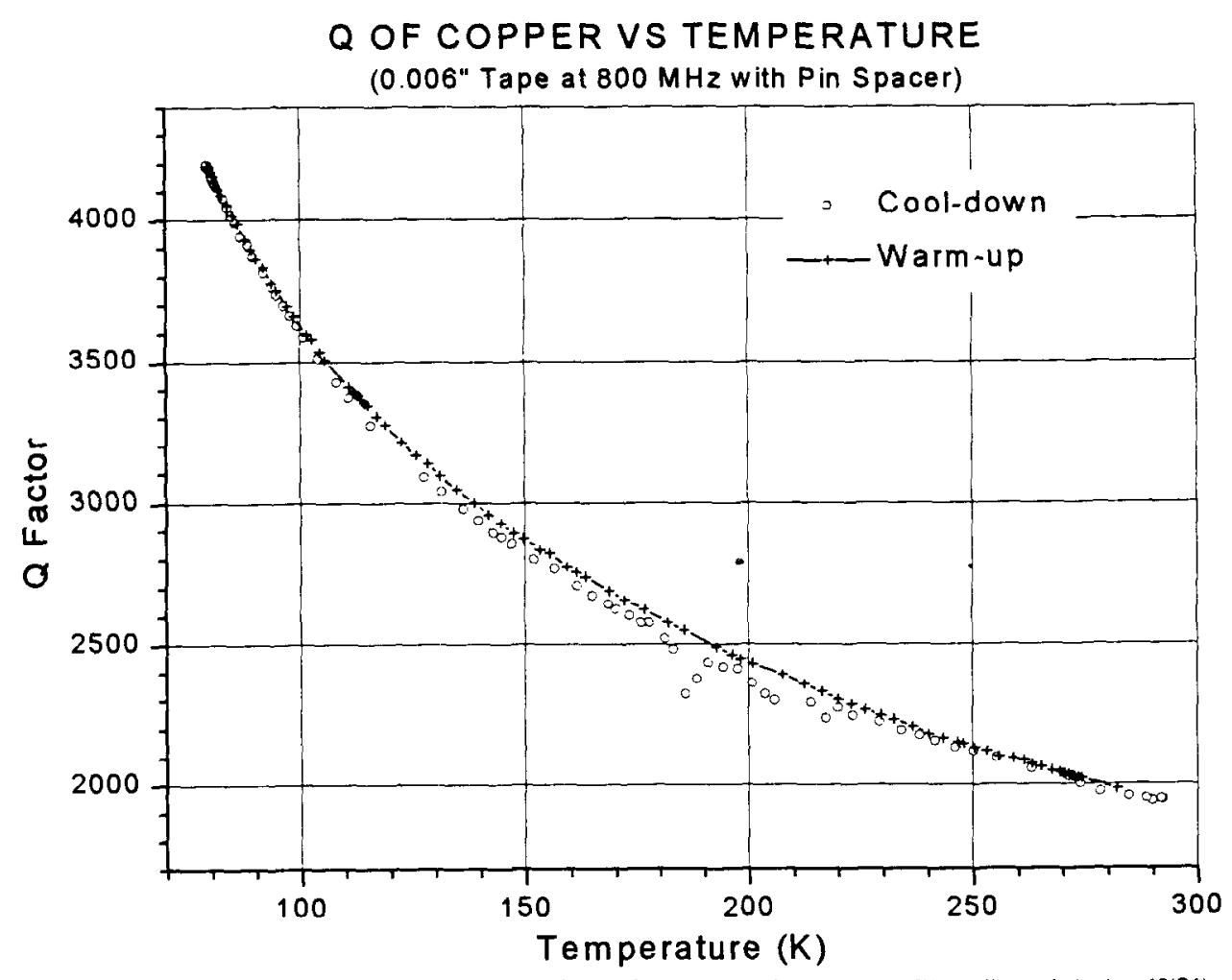

Fig. 16 Copper $Q$ factor (with pin spacer)

Q OF COPPER VS TEMPERATURE

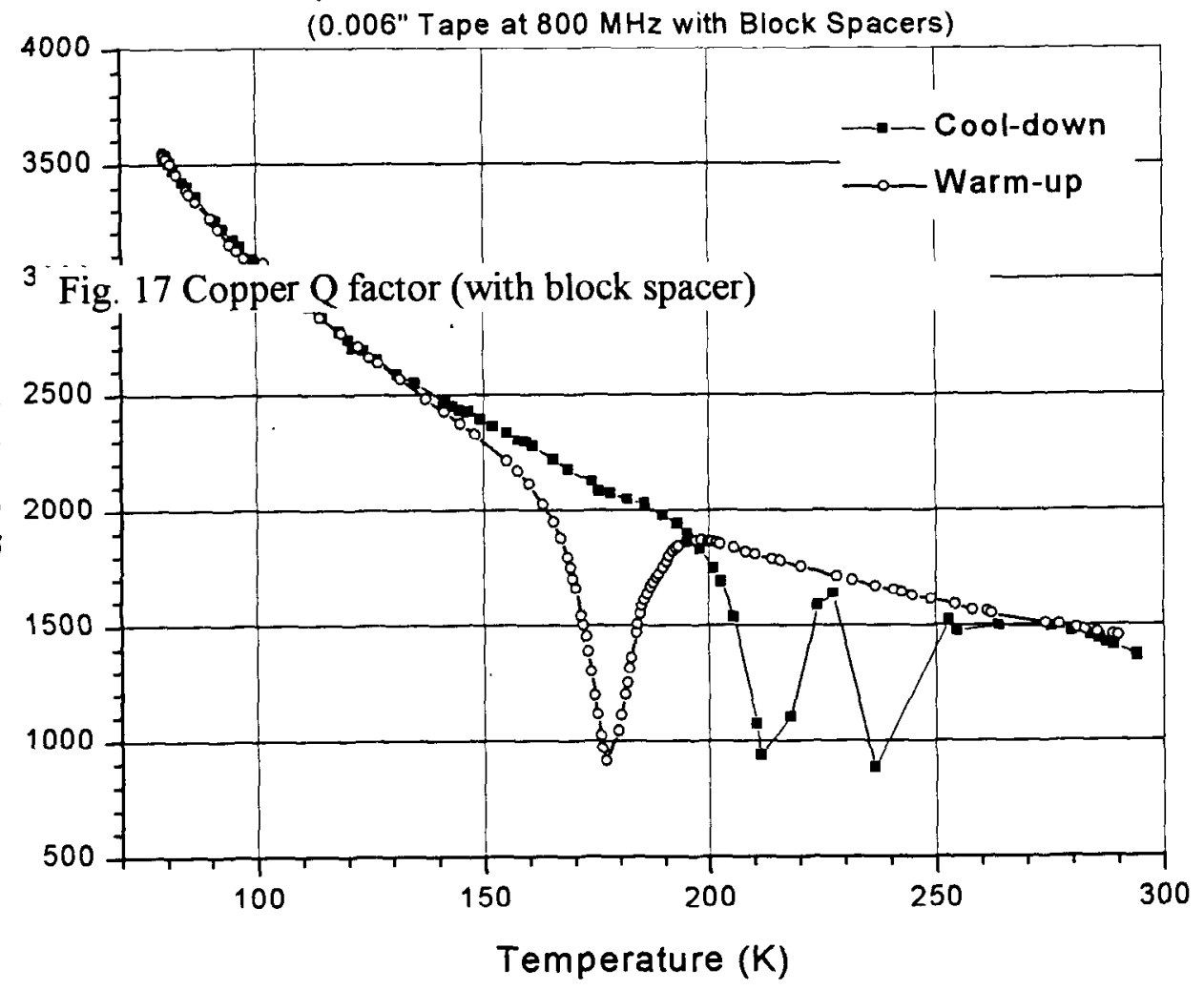

Fig. 17 Copper Q factor (with block spacer) 


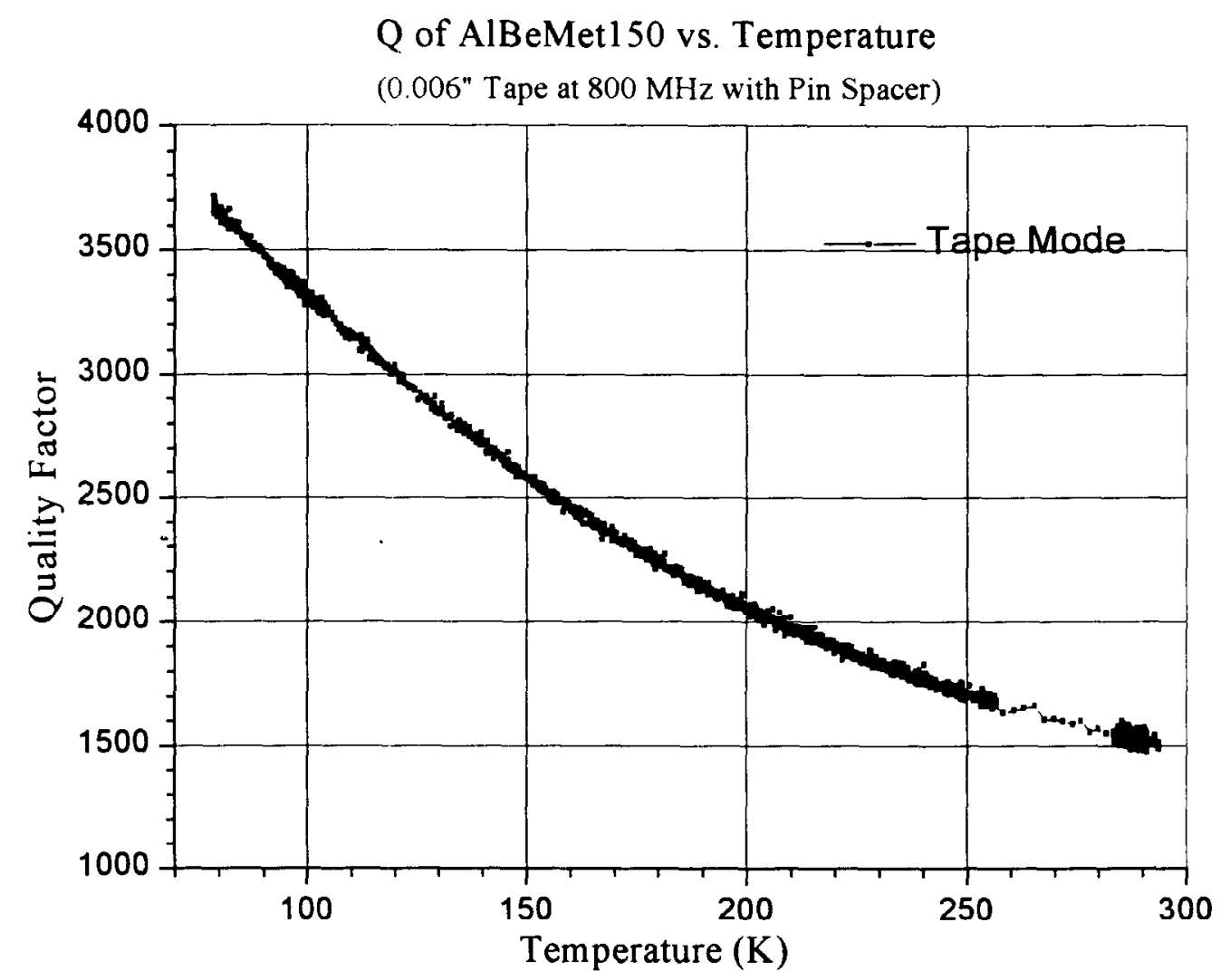

Fig. $18 \mathrm{Q}$ factor of AlBeMet 150 (with pin spacer)

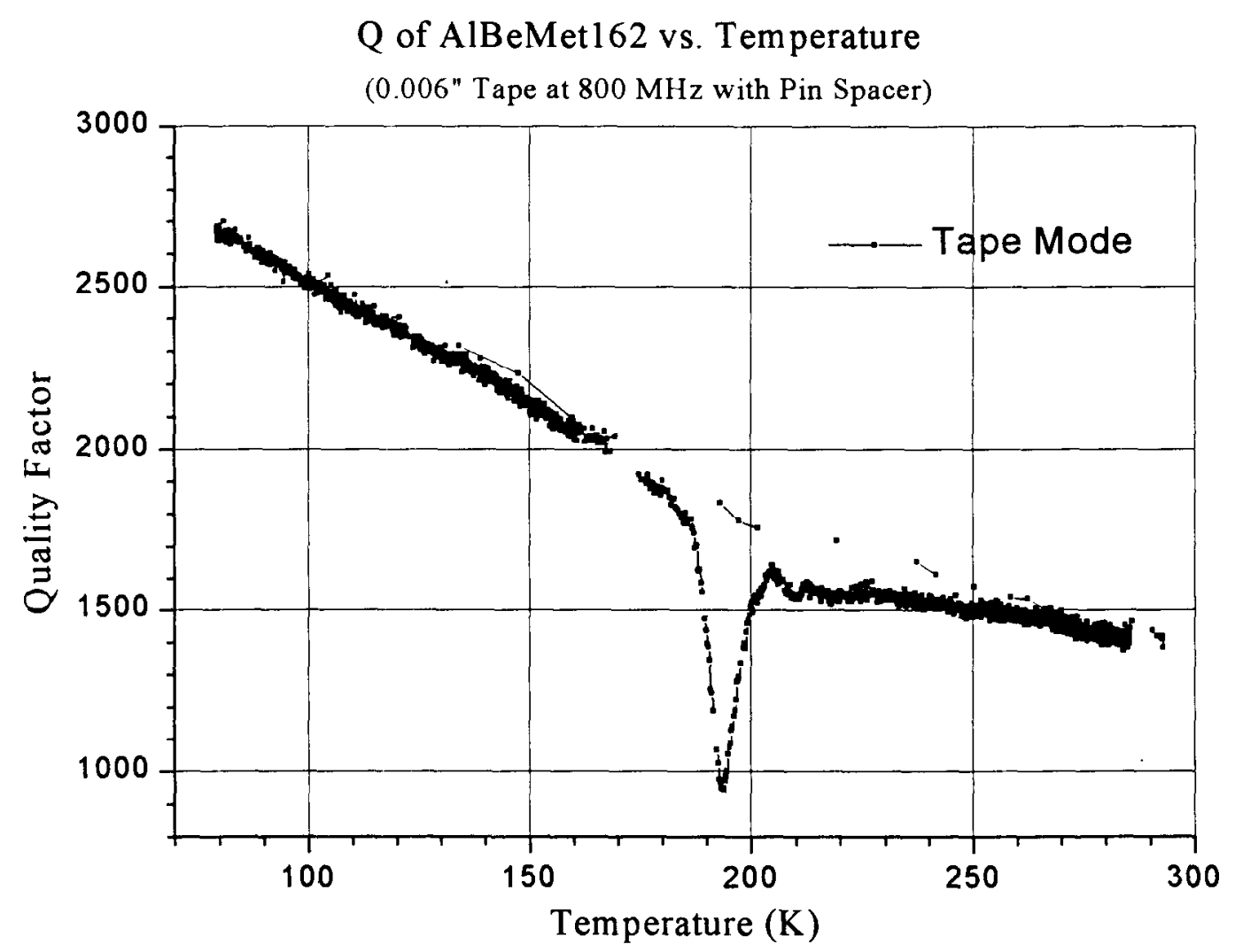

Fig. $19 \mathrm{Q}$ factor of AlBeMet 162 (with pin spacer) 
It was observed that the TM1 10 mode frequency varied in a wide range and was sensitive with temperature and mechanical assembly. Besides, its $Q$ was usually pretty low, around 200, and changed with temperature too. In the case of block spacer, we found its frequency could be so close to the tape frequency that makes two modes coupled each other. This caused the resonant curve of tape mode being seriously deformed. The process of the network analyzer to figure out $\mathrm{Q}$ factor is based on the measurement of $3 \mathrm{~dB}$ bandwidth, which is no longer correct when the resonant curve is deformed. Thus the data collected were wrong. This is the main reason why the curve shown in Fig. 17 is not smooth. One can see from above table, in case of block spacer the tape mode frequency reduces to $840 \mathrm{MHz}$ from $890 \mathrm{MHz}$ meaning mode interference is easier to occur. Indeed the curves with pin spacer are much smoother than with block spacer.

Figs. 18 and 19 show the results of aluminium-beryllium alloy AlBEMet-150 and AlBeMet 162 respectively. One can see that their conductivities are close to those of beryllium at room temperature, but worse than beryllium at $\mathrm{LN}_{2}$ temperature.

\section{DATA ANAL YSES}

It is well known that $Q$ factor of a cavity is proportional to the metal rf surface conductivity, which is proportional to the square root of dc volume conductivity. Since the tape resonator involves dielectric, of which the loss is unknown but we cannot ignore, how to determine the conductivity is much complex.

The $Q$ factor can be expressed by two terms with the following formula:

$$
\frac{1}{Q_{L}}=\frac{1}{Q_{m}}+\frac{1}{Q_{d}}
$$

where $Q_{L}$, i.e. loaded $Q$, is only parameter can be measured, $Q_{m}$ and $Q_{d}$ refer to the losses due to metal and dielectric respectively.

The issue is how to determine $Q_{m}$ and $Q_{d}$ when only $Q_{L}$ is measured. It is more convenient to use decay coefficient, which is the reciprocal of $Q$ factor, as follows:

$$
\delta_{L}=\delta_{m}+\delta_{d}
$$

where subscripts ${ }_{-\mathrm{m},-\mathrm{d},-\mathrm{L}}$ refer to metal, dielectric and total loaded. $\delta_{L}=1 / Q_{L}$ is only parameter can be measured directly. Nevertheless, from MAFIA simulation we can get some useful information for the others. Table 6 lists the $\mathrm{Q}$ factors of beryllium from previous section, and the $\delta$ s calculated accordingly.

Table 6

\begin{tabular}{|c|c|c|c|c|c|c|}
\hline \multirow{3}{*}{ Spacer } & \multicolumn{3}{|c|}{$Q^{\text {Factor }}$} & \multicolumn{3}{c|}{ Decay coefficient } \\
\cline { 2 - 7 } & Total & Metal & Dielectric & Total & Metal & Dielectric \\
\cline { 2 - 7 } & $Q_{L}$ & $Q_{m}$ & $Q_{d}$ & $\delta\left(\times 1 \sigma^{-4}\right)$ & $\delta_{m}\left(\times 1 \sigma^{-4}\right)$ & $\delta_{d}\left(\times 1 \sigma^{-4}\right)$ \\
\hline Pin & 2968 & 3520 & 18762 & 3.369 & 2.841 & 0.528 \\
\hline Block & 1871 & 3408 & 4148 & 5.345 & 2.934 & 2.411 \\
\hline
\end{tabular}

$\left(Q_{L}\right.$ and $Q_{m}$ from Tables $3 \& 4, \delta \& \delta_{m}$ obtained from their reciprocal, $\delta_{d}=\delta-\delta_{m}$ and $\left.Q_{d}=1 / \delta_{d}\right)$ 
Then we get following two equations

$$
\begin{aligned}
& \delta_{p}=\delta_{m p}+\delta_{d p} \quad \text { where } \delta_{m p}=2.841 \times 10^{-4}, \delta_{d p}=0.528 \times 10^{-4} \\
& \delta_{b}=\delta_{m b}+\delta_{d b} \quad \delta_{m b}=2.934 \times 10^{-4}, \delta_{d b}=2.411 \times 10^{-4}
\end{aligned}
$$

where subscripts ${ }_{-.}$refer to pin spacer and block spacer respectively. Remember that they are deduced from MAFIA simulation in the conditions that: (1) metal conductivity $K=5.8 \times 10^{7}$ $m h o / m$, (2) dielectric loss tangent $\tan \delta=0.001$. (3) The mesh chosen is coarse.

Note also that the metal losses $\delta_{m p}$ and $\delta_{m b}$ have minor difference because the frequencies are different.

In the experiment, both metallic conductivity and dielectric loss tangent are uncertain. So we have to put correct coefficient on those $\delta$ parameters. There are three coefficients, $C_{B e} C_{C u} C_{d}$, referring to beryllium, copper and dielectric respectively. Two pairs of measurements for copper and beryllium were made as shown on Figs. 14 to 17. Besides, we realized that the data based on a coarse mesh in MAFIA code are not precise, additional correct coefficient, $\alpha_{m}$, is necessary to put on the metallic loss term $\delta_{m p}$ and $\delta_{m b}$. Plausibly, we assume $\alpha_{m}=1.63$ in accordance with Reference [15]. Thus we got four equations:

$$
\begin{aligned}
& \delta_{B e p}=C_{B e} \alpha_{m} \delta_{m p}+C_{d} \delta_{d p} \\
& \delta_{B e b}=C_{B e} \alpha_{m} \delta_{m b}+C_{d} \delta_{d b} \\
& \delta_{C u p}=C_{C u} \alpha_{m} \delta_{m p}+C_{d} \delta_{d p} \\
& \delta_{C u b}=C_{C u} \alpha_{m} \delta_{m b}+C_{d} \delta_{d b}
\end{aligned}
$$

The four $\delta \mathrm{s}$ (the reciprocal of $\mathrm{Q}$ ) on the left hand side are determined by experiments for each temperature. The $\delta$ parameters on right hand side are determined by (6); the three correction coefficients $C$ are to be determined. In principle, four equations are more than adequate to determine three unknowns, but those equations are not independent. From (7), one can deduces the following equation:

$$
\frac{\delta_{C u p}-\delta_{B e p}}{\delta_{m p}}=\frac{\delta_{C u b}-\delta_{B e b}}{\delta_{m b}}
$$

The measured data should be consistent with equation (8). Taking the error of experimental data into account, on should find a best fitting. However, to fit above data is rather complex. From preceding section, Table 7 lists the measured $\mathrm{Q}$ factors. Also shown are $\delta \mathrm{s}$ accordingly.

Table 7

\begin{tabular}{|c|c|c|c|c|c|c|}
\hline \multirow{2}{*}{ Material } & \multirow{2}{*}{ Spacer } & \multicolumn{2}{|c|}{$\mathrm{Q}$} & & \multicolumn{2}{c|}{$\delta\left(10^{-4}\right)$} \\
\cline { 7 - 8 } & & Room Temp & $80 \mathrm{k}$ & & Room Temp & $80 \mathrm{k}$ \\
\hline $\mathrm{Be}$ & Pin & $1375 \sim 1420$ & $4189 \sim 4203$ & $\delta_{p B e}$ & $7.04 \sim 7.41$ & $2.38 \sim 2.39$ \\
\hline $\mathrm{Be}$ & Block & $1112 \sim 1140$ & $3482 \sim 3486$ & $\delta_{B B}$ & $8.77 \sim 8.99$ & $2.87 \sim 2.87$ \\
\hline $\mathrm{Cu}$ & Pin & $1944 \sim 1945$ & $4149 \sim 4155$ & $\delta_{p C u}$ & $5.14 \sim 5.14$ & $2.41 \sim 2.41$ \\
\hline $\mathrm{Cu}$ & Block & $1364 \sim 1451$ & $3527 \sim 3534$ & $\delta_{B C u}$ & $6.89 \sim 7.33$ & $2.83 \sim 2.84$ \\
\hline
\end{tabular}


After deliberate algebraic manipulation, we got the best fitting to (7) listing in Table 8.

Table 8

\begin{tabular}{|c|c|c|}
\hline & Room Temp & $80 \mathrm{~K}$ \\
\hline$C_{B e}$ & 1.44 & 0.49 \\
\hline$C_{C u}$ & 1.01 & 0.49 \\
\hline$C_{d}$ & 0.88 & 0.20 \\
\hline
\end{tabular}

These data can convert to the resistivity or tangent angle via the following formulae:

$$
\begin{aligned}
& \text { Metal Resistivity }=C_{m}{ }^{2} / 5.8 \times 10^{7} \quad(\mathrm{Ohm}-\mathrm{m}) \\
& \text { Dielectric Tangent }=C_{d} \times 0.001
\end{aligned}
$$

Finally, Table 9 summarizes the results.

Table 9

\begin{tabular}{|c|c|c|c|c|}
\hline \multirow{2}{*}{ Material } & \multirow{2}{*}{ Parameter } & \multirow{2}{*}{ Unit } & \multicolumn{2}{|c|}{ Temperature } \\
\cline { 4 - 5 } & & & Room Temp & $80 \mathrm{~K}$ \\
\hline Beryllium & Resistivity & $10^{-8}(\mathrm{Ohm}-\mathrm{m})$ & 3.6 & 0.41 \\
\hline Copper & Resistivity & $10^{-8}(\mathrm{Ohm}-\mathrm{m})$ & 1.76 & 0.41 \\
\hline Rexolite & Tangent angle & 1 & 0.0009 & 0.0002 \\
\hline
\end{tabular}

At room temperature, the data are coincident with those from handbook, but at liquid nitrogen temperature, they are not. Nevertheless, we found at $\mathrm{LN}_{2}$ temperature the resistivity for beryllium is very close to that of copper.

Based on the correct coefficient of Rexolite $C_{d}$ found above, one could also find the resistivity of aluminium-beryllium alloy. Figs. 18 and 19 show the measured $Q$ factors of ALBeMet150 and ALBeMet162 respectively. Both are with a pin spacer. One can apply the formula:

$$
\begin{aligned}
\delta_{m} & =C_{m} \alpha_{m} \delta_{m p}+C_{d} \delta_{d p} \\
\text { or } \quad C_{m} & =\frac{\delta_{m}-C_{d} \delta_{d p}}{\alpha_{m} \delta_{m p}}
\end{aligned}
$$

where $\delta_{m}$ is the metallic delay coefficient, $\delta_{m}=1 / Q$. Substituting measured $Q$, one can find the coefficient and resistivity accordingly. Table 10 summarizes the results.

Table 10

\begin{tabular}{|c|c|c|c|c|}
\hline \multirow{2}{*}{ Material } & \multicolumn{2}{|c|}{ Coefficient } & \multicolumn{2}{c|}{ Resistivity (10-8 Ohm-m) } \\
\cline { 2 - 5 } & Room Temp & $80 \mathrm{~K}$ & Room Temp & $80 \mathrm{~K}$ \\
\hline AlBeMet-150 & 1.34 & 0.56 & 3.1 & 0.54 \\
\hline AlBeMet-162 & 1.44 & 0.79 & 3.6 & 1.1 \\
\hline
\end{tabular}




\section{ACKNOWLEDGEMENTS}

This work was supported by the US DOE under Contract No. DE-AC02-98CH10886. Thanks also to all the members in CAP group and muon collider community who encouraged this work. Many of them gave valuable comments and suggestions and reviewed this report as well. We are especially indebted to many colleagues Labwide who helped a lot in all respects to make this experiment realizable, among them are L. Jia, V. LoDestro, L. Addessi, L. Snydstrup, G. Miglionico, M. McNeill, T. Lelle, R. Bowman, X. J. Wang and many others.

\section{Referrences}

[1] R. Palmer, A. Tollestrup and A. Sessler, "Status Report of a High Luminosity Muon Collider and Future Research and Development Plans". BNL-63865, CAP-154-MUON-96C, Nov., 1996.

[2] Y. Zhao, "Liquid Nitrogen Cooling for Accelerator RF", Muon Collider Workshop, Rosario, Washington, May 17-20,1997.

[3] CRC Handbook of Chemistry and Physics, 77th Edition, 1996-1997

[4] L. A. Hall, "Survey of Electrical Resistivity Measurements on 16 pure Metals in the Temperature range 0 to $273^{\circ} \mathrm{K}$ ", NBS Tech. Note 365, Feb. 1968.

[5] T. C. Chi, "Electrical resistivity of alkaline earth elements", J. Phys Chem. Ref. Data, Vol.8, No.2, 1979.

[6] Los Alamos National Laboratory, Memorandum "Beryllium cavity test - status report", Sep. 16,1991 .

[7] WADD Tech. Report 60-56.

[8] H. B.G. Casimir, "The skin effect', Philips Tech. Rev. V.28, no.10, p.300, 1967.

[9] G. Bolme "Cryogenic surface studies", LANL memo. AT-2:88-134, 1988

[10] W. Chou, F. Ruggiero, “Anomalous skin effect and resistive wall heating", LHC project Note 2.(1995).

[11] A. B. Pippard, "Metallic conduction at high frequency and low temperature", Advances in Electronics \& Electro Physics, Vol.6, 1, 1954, p.22.

[12] Reuter, G. E.H., and Sondheimer, E.H., Proc. Roy. Soc. (London) A195, 336, (1948)

[13] Y. Zhao, H. Wang, "Experiment of electrical conductivity at low temperature (preliminary measurement)", BNL-65629, CAP-221-MUON-98R, July, 1998.

[14] Y. Zhao, "Beryllium properties measurements at low temperature', Presentation on LBNL Muon-RF workshop, Feb, 18-20, 1999

[15] Y. Zhao, H. Wang, "A study of the edge field effect using MAFIA simulation and conformal mapping", BNL-66930, CAP-262-Muon-99R, 1999.

[16] GoodFellow Catalog 1998/1999 